\title{
A REMARK ON THE CONTINUITY OF THE DUAL PROCESS
}

\author{
MAMORU KANDA
}

\section{§ 1. Introduction}

Let $S$ be a locally compact (not compact) Hausdorff space satisfying the second axiom of countability and let $\mathscr{B}$ be the $\sigma$-field of all Borel subsets of $S$ and let $\mathscr{A}$ be the $\sigma$-field of all the subsets of $S$ which, for each finite measure $\mu$ defined on $(S, \mathscr{A})$, are in the completed $\sigma$-field of $\mathscr{B}$ relative to $\mu$. We denote by $C_{0}$ the Banach space of continuous functions vanishing at infinity with the uniform norm and $B_{k}$ the space of bounded $\mathscr{A}$-measurable functions with compact support in $S$.

Let $X=\left(x_{t}, \zeta, M_{t}, P_{x}\right)$ be a standard process ${ }^{1)}$ on $S$. Let us set

$$
G_{\alpha}(x, B)=\int_{0}^{+\infty} e^{-\alpha t} P_{x}\left(x_{t} \in B\right) d t, \quad \alpha \geqq 0,
$$

where $B$ is a $\mathscr{A}$-measurable set and set $G_{\alpha} f(x)=\int_{S} f(y) G_{\alpha}(x, d y)$ for each bounded $\mathscr{A}$-measurable function $f$. We say that the standard process $X$ satisfies the regularity condition with respect to a locally finite measure $m(d x)$, if the following holds :

(i) $G_{0}(x, K)$ is bounded on every compact set when $K$ is compact.

(ii) $G_{\alpha}(x, K)$ is absolutely continuous with respect to $m(d x)$ for each $\alpha \geq 0$ and for each $x \in S$.

(iii) $G_{\alpha} f(x)$ is finite and contiuous for each $f \in B_{k}$.

We say that two standard processes on $S X=\left(x_{t}, \zeta, M_{t}, P_{x}\right)$ and $\hat{X}=\left(\hat{x}_{t}, \hat{\zeta}, \hat{M}_{t}, \hat{P}_{x}\right)$ are in the relation of duality with respect to a locally finite measure $m(d x)$, if each of them satisfies the regularity condition with respect to $m(d x)$ and it holds that for each $\alpha \geqq 0$

$$
\int_{S} g(x) G_{\alpha} f(x) m(d x)=\int_{S} f(x) \hat{G}_{\alpha} g(x) m(d x), \quad f, g \in B_{k},
$$

Received August 25, 1967.

Revised September 18, 1967.

1) For the definition see H. Kunita and T. Watanabe [3]. 
where

$$
\hat{G}_{\alpha}(x, B)=\int_{0}^{+\infty} e^{-\alpha t} \hat{P}_{x}\left(\hat{x}_{t} \in B\right) d t, \quad \hat{G}_{\alpha} f(x)=\int_{S} f(y) \hat{G}_{\alpha}(x, d y) .
$$

Our aim is to show the following theorem.

Theorem. Let $X$ and $\hat{X}$ be standard processes on $S$ in the relation of duality with respect to a locally finite measure $m(d x)$. Further let us suppose that the semi-group $\left\{T_{t}\right\}_{t \geq 0}$ of $X$ and $\left\{\hat{T}_{t}\right\}_{t \geq 0}$ of $\hat{X}$ are strongly continuous operators on $C_{0}$. Then the process $\hat{X}$ is a continuous process, if and only if $X$ is $a$ continuous process.

In the author's previous paper [2], we studied the process $X$ connected with the following strictly elliptic differential operator on the ball $\Omega$ of $R^{d} \quad(d \geq 3)$

$$
D=\sum_{i, j=1}^{d} a_{i j}(x) \frac{\partial^{2}}{\partial x_{i} \partial x_{j}}+\sum_{i=1}^{d} a_{i}(x) \frac{\partial}{\partial x_{i}}
$$

and the process $\hat{X}$ connected with the formal adjoint operator

$$
\hat{D}=\sum_{i \cdot j=1}^{d} \frac{\partial^{2}}{\partial x_{i} \partial x_{j}}\left(a_{i j}(x) \cdot\right)-\sum_{i=1}^{d} \frac{\partial}{\partial x_{i}}\left(a_{i}(x) \cdot\right),
$$

where $D$ is assumed to satisfy the condition $(L)$, that is,

$$
-\int_{\Omega} D v(x) d x \geq 0
$$

holds for every non-negative $C^{2}$-functions $v$ with compact support in $\Omega$ and the coefficients $\left\{a_{i j}, i \cdot j=1,2, \cdots, d\right\}$ and $\left\{a_{i} ; i=1,2, \cdots, d\right\}$ are bounded and uniformly Hölder continuous such that $a_{i j}=a_{j i}$.

By using the above theorem and the proposition in $\S 4$, we can show the following

Corollary. The process $\hat{X}$ connected with the operator $\hat{D}$ which is mentioned above is a continuous process.

\section{\& 2. Resolvent kernels.}

Throughout this section we use the notations in H. Kunita and T. Watanabe [3].

A function $R_{\alpha}(x, A)$, defined for $\alpha>O, x$ of $S$ and $A$ of $\mathscr{A}$, is said to be a resolvent kernel if it satisfies the following conditions (a)-(d). (a). 
For each $\alpha>O$ and $x$ of $S, R_{\alpha}(x, \cdot)$ is a locally finite measure. (b). Let $f$ be a bounded $\mathscr{A}$-measurable function of compact support, then $R_{\alpha} f$ is $\mathscr{A}$-measurable and bounded on every compact set, where we write $R_{\alpha} f$ for $\int f(y) R_{\alpha}(\cdot, d y)$. (c). The resolvent equation $R_{\alpha} f-R_{\beta} f+(\alpha-\beta) R_{\alpha} R_{\beta} f$ $=O$ is satisfied and (d). $\lim _{\alpha \rightarrow+\infty} R_{\star} f(x)=O$ for each $x$ and for each bounded $\mathscr{A}$-measurable function $f$ of compact support.

Since $R_{\alpha}(x, A) \geq R_{\beta}(x, A)$ for each $A$ of $\mathscr{A}$

$$
R_{0}(x, A)=\lim _{\alpha \rightarrow 0} R_{\alpha}(x, A)
$$

exists for each $A$ of $\mathscr{A}$ and defines a measure on $\mathscr{A}$.

Let $\left\{R_{\alpha}(x, A)\right\}$ be a resolvent kernel and $m$, a measure defined over $(S, \mathscr{A})$.

$\left\{R_{\alpha}(x, A)\right\}$ is said to be dominated by $m$ if, for each $\alpha>0, R_{\alpha}(x, A)$ satisfies the condition (ii) of $\S 1$.

$\left\{R_{\alpha}(x, A)\right\}$ is said to be integrable if $R_{0}(\cdot, A)$ satisfies the condition (i) of $\S 1$.

$\left\{R_{\alpha}(x, A)\right\}$ is said to be regular if, for each continuous function $f$ of compact support, $\alpha R_{\alpha} f$ converges boundedly on every compact set to $f$ as $\alpha \rightarrow+\infty$.

A resolvent kernel $\left\{\hat{R}_{\alpha}(x, A)\right\}$ is called the co-resolvent kernel of $\left\{R_{\alpha}(x, A)\right\}$ with respect to $m(d x)$ if, for each $f, g$ of $B_{k}$ and for each $\alpha>0$

$$
\int_{S} f(x) R_{\alpha} g(x) d x=\int_{S} g(x) \hat{R}_{\alpha} f(x) d x .
$$

A non-negative $\mathscr{A}$-measurable function $u$ is said to be $(R, \alpha)$-excessive if $\beta R_{\alpha+\beta} u \leqq u$ for all $\beta>0$ and if $\lim _{\beta \rightarrow+\infty} \beta R_{\alpha+\beta} u=u$.

Given a number $\alpha \geqq O$, a jointly $(=\mathscr{A} \times \mathscr{A})$ measurable function $R_{\alpha}(x, y)$ is said to be the potential kernel of exponent $\alpha$ if the following conditions are satisfied : (a) $R_{\alpha}(x, d y)=R_{\alpha}(x, y) m(d y)$; (b) $\hat{R}_{\alpha}(y, d x)=R_{\alpha}(x, y) m(d x)$; (c) $R_{\alpha}(\cdot, y)$ is $(R, \alpha)$-excessive for each fixed $y$ and $(\mathbf{d}) R_{\alpha}(x, \cdot)$ is $(\hat{R}, \alpha)$ excessive for each fixed $x$.

The following lemma is Theorem 1 in H. Kunita and T. Watanabe [3].

Lemma 1. (H. Kunita and T. Watanabe). Let $\left\{R_{\alpha}(x, A)\right\}$ be a resolvent kernel and $\left\{\hat{R}_{\alpha}(x, A)\right\}$ be the co-resolvent kernel of $\left\{R_{\alpha}(x, A)\right\}$. Assume that 
$\left\{R_{\alpha}(x, A)\right\}$ and $\left\{\hat{R}_{\dot{u}}(x, A)\right\}$ are dominated by the locally finite measure $m(d x)$. Then there is a unique potential kernel of exponent $\alpha$ for $\alpha \geqq O$.

\section{§ 3. Fundamental lemmas.}

Throughout this section we treat two standard processes on $S X$ $=\left(x_{t}, \zeta, M_{t}, P_{t}\right)$ and $\hat{X}=\left(\hat{x}_{t}, \hat{\zeta}, \hat{M}_{t}, \hat{P}_{x}\right)$ which are in the relation of duality with respect to a locally finite measure $m(d x)$ without special mentioning.

Evidently $\left\{G_{\alpha}(x, A)\right\}$ is a resolvent kernel and $\left\{\hat{G}_{\alpha}(x, A)\right\}$ is the coresolvent kernel which are dominated by $m(d x)$ by the condition (ii) in $\S 1$. Hence the following lemma is a direct consequence of lemma 1 .

Lemma 2. There is a unique potential kernel $G_{\alpha}(x, y)$ of exponent $\alpha$ for all $\alpha \geqq 0$.

Let $E$ be an analytic set in $S$ and let us set $\sigma_{E}=i n f\left(t>0, x_{t} \in E\right)$, $=+\infty$ if the set $\left(t>O, x_{t} \in E\right)$ is empty.

The next lemma plays an essential role in this paper, which is first shown by G.A. Hunt [1] under his assumptions $(F)$ and $(G)$ and P.A. Meyer [4] has next shown it under a little different assumption. Our case follows directly from P.A. Meyer's result.

Lemma 3. Suppose that the semi-groups $\left\{T_{t}\right\}_{t \geq 0}$ and $\left\{\hat{T}_{t}\right\}_{t \geq 0}$ of the processes $X$ and $\hat{X}$ respectively are the strongly continuous operators on $C_{0}$. Then, for each analytic set $E$ in $S$, it holds that

$$
\int_{S} G_{0}(x, z) \hat{P}_{y}\left(\hat{x}_{\sigma_{E}} \in d z\right)=\int_{S} G_{0}(z, y) P_{x}\left(x_{\sigma_{E}} \in d z\right)
$$

for eaxch $x$ and $y$ in $S$.

Proof. Let us note that the notion " $(G, \alpha)$-excessive" is equivalent to the notion " $\alpha$-excessive with respect to $\left\{T_{t}{ }^{\prime}{ }^{2)}\right.$. Then the semi-group $\left\{T_{t}\right\}$ of $X$ and $\left\{\hat{T}_{t}\right\}$ of $\hat{X}$ are in the relation of duality in Meyer' $s$ sense by Lemma 2. Therefore, for each $\alpha>0$ it holds that

$$
\int_{S} G_{\alpha}(z, y) P_{E}^{\alpha}(x, d z)=\int_{S} G_{\alpha}(x, z) \hat{P}_{E}^{\alpha}(y, d z),
$$

where $P_{E}^{\alpha}(x, d z)=E_{x}\left(e^{-\alpha \sigma_{E}} ; x_{\sigma_{E}} \in d z\right), \hat{P}_{E}^{\alpha}(y, d z)=\hat{E}_{y}\left(e^{-\alpha \sigma_{z}} ; \hat{x}_{\sigma_{E}} \in d z\right)$. Noting that $\lim _{\alpha \rightarrow 0} G_{\alpha}(x, y) \uparrow G_{0}(x, y)$, we have

2) We say that a non-negative $\mathscr{A}$-measurable function $u(x)$ is $\alpha$-excessive with respect to $\left\{T_{t}\right\}$, if $E_{x}\left(e^{-\alpha t} u\left(x_{t}\right), t<\zeta\right) \leqq u(x)$ for each $t>0$ and $\lim _{t \rightarrow 0} E_{\alpha}\left(t^{-\alpha t} u\left(x_{t}\right), t<\zeta\right)=u(x)$. 
(1)

$$
\begin{aligned}
& \int_{S} G_{\alpha}(z, y) P_{E}^{\alpha}(x, d z) \leqq \int_{S} G_{0}(z, y) P_{x}\left(x_{\sigma_{E}} \in d z\right), \\
& \int_{S} G_{\alpha}(x, z) \hat{P}_{E}^{\alpha}(y, d z) \leqq \int_{S} G_{0}(x, z) P_{y}\left(\hat{x}_{\sigma_{E}} \in d z\right)
\end{aligned}
$$

On the other hand, we have for each fixed $\beta>0$

$$
\begin{aligned}
& \int_{S} G_{0}(z, y) P_{E}^{\beta}(x, d z)=\lim _{\alpha \rightarrow 0} \int_{S} G_{\alpha}(z, y) P_{E}^{\beta}(x, d z) \leqq \lim _{\alpha \rightarrow 0} \int_{S} G_{\alpha}(z, y) P_{E}^{\alpha}(x, d z), \\
& \int_{S} G_{0}(x, z) \hat{P}_{E}^{\beta}(y, d z)=\lim _{\alpha \rightarrow 0} \int_{S} G_{\alpha}(x, z) \hat{P}_{E}^{\beta}(y, d z) \leqq \lim _{\alpha \rightarrow 0} \int_{S} G_{\alpha}(x, z) \hat{P}_{E}^{\alpha}(y, d z) .
\end{aligned}
$$

Hence by tending $\beta$ to zero we can show that

$$
\begin{aligned}
& \int_{S} G_{0}(z, y) P_{x}\left(x_{\sigma_{E}} \in d z\right) \leqq \lim _{\alpha \rightarrow 0} \int_{S} G_{\alpha}(z, y) P_{E}^{\alpha}(x, d z) \\
& \int_{S} G_{0}(x, z) \hat{P}_{y}\left(x_{\sigma_{E}} \in d z\right) \leqq \lim _{\alpha \rightarrow 0} \int_{S} G_{\alpha}(x, z) \hat{P}_{E}^{\alpha}(y, d z) .
\end{aligned}
$$

From the inegqualities (1) and (2) we can prove the lemma.

Now, let us note that $\left\{G_{\alpha}(x, A)\right\}$ and $\left\{\hat{G}_{\alpha}(x, A)\right\}$ satisfy the hypothesis $(B)$ in H. Kunita and T. Watanabe [3], that is, $G_{\alpha}(x, A)$ is integrable and dominated by a locally finite measure $m(d x),\left\{\hat{G}_{\alpha}(x, A)\right\}$ is regular and $\hat{G}_{\alpha} f, \alpha \geqq O$ is continuous and finite everywhere for each $f$ of $B_{K}$. Then, by Theorem 7 in H. Kunita and T. Watanabe [3], Proposition 7. 11 in [3] is valid for the processes $X$ and $\hat{X}$. Hence we have the following

Lemma 4. If the measures $\mu_{1}$ and $\mu_{2}$ define the same potential, i.e. $\int_{S} G_{0}(x, y) \mu_{1}(d y)=\int_{S} G_{0}(x, y) \mu_{2}(d y)$, which is integrable over each compact set, then we have $\mu_{1}=\mu_{2}$.

\section{$\S 4$. Proof of the Theorem.}

In this section we always treat the processes $X$ and $\hat{X}$ mentioned in the Theorem.

Let us assume that $X$ is a continuous process. Since $m(d x)$ can be considered as a reference measure by the regularity condition, according to Corollary to Theorem 4.2 in S. Watanabe [5], for the proof of the continuity of the process $X$, we have only to show that

3) $\{\infty\}$ is adjoined to $S$ and $S \cup\{\infty\}$ denotes the one-point compactification of $S$. For each function $f$ we set $f(\{\infty\}=0$. 


$$
\hat{P}_{x}\left(\hat{x} \hat{\sigma}_{Q^{c}} \in \partial Q \cup\{\infty\} ; \hat{\sigma}_{Q^{c}}<+\infty\right)=\hat{P}_{x}\left(\hat{\sigma}_{Q^{c}}<+\infty\right), x \in Q
$$

for a bounded and non-empty open set $Q$, where $Q^{c}=S-Q$. We shall first prove the following equality

$$
\int_{S} G_{0}\left(z, x_{0}\right) P_{x}\left(x_{\sigma_{Q^{c}}} \in d z\right)=\int_{S} G_{0}\left(z, x_{0}\right) P_{x}\left(x_{\sigma_{\partial Q}} \in d z\right)
$$

for each $x$ and $x_{0} \in Q$. When $x$ is in the interior of $Q$, the equality (4) holds by the continuity of the path of $X$. In case $x \in \bar{Q}^{c} \cup \partial Q^{r e g}$, where $\bar{Q}$ denotes the closure of $Q$ and $\partial Q^{r e g}$ denotes the set of all regular points of $\partial Q$ for $X$, the lef-hand side of (4) equals to $G_{0}\left(x, x_{0}\right)$. For $x \in \partial Q^{r e g}$ it is clear that the right-hand side of (4) equals $G_{0}\left(x, x_{0}\right)$ too. When $x \in \bar{Q}^{c}$, by the continuity of the path we have

$$
\int_{S} G_{0}\left(z, x_{0}\right) P_{x}\left(x_{\sigma_{\partial Q}} \in d z\right)=\int_{S} G_{0}\left(z, x_{0}\right) P_{x}\left(x_{\sigma_{\bar{Q}}} \in d z\right),
$$

and by lemma 3

$$
\int_{S} G_{0}\left(z, x_{0}\right) P_{x}\left(x_{\sigma_{\bar{Q}}} \in d z\right)=\int_{S} G_{0}(x, z) \hat{P}_{x_{0}}\left(\hat{x}_{\bar{Q}} \in d z\right) .
$$

Noting that $P_{x_{0}}\left(x_{\sigma_{\bar{Q}}} \in d z\right)=\delta_{x_{0}}(d z)$, where $\delta_{x_{0}}(d z)$ is the Dirac measure at $x_{0}$, we have

$$
\int_{S} G_{0}\left(z, x_{0}\right) P_{x}\left(x_{\sigma_{\partial Q}} \in d z\right)=G_{0}\left(x, x_{0}\right) .
$$

Hence the equality (4) holds on $S-\left(\partial Q-\partial Q^{r e g}\right)$. On the other hand we have $m\left(\partial Q-\partial Q^{r e g}\right)=0$. Indeed, $G_{0}\left(x, \partial Q-\partial Q^{r e g}\right)=0$ for each $x \in S$, because $\partial Q-\partial Q^{r e g}$ is a semi-polar set, therefore $G_{\alpha}\left(x, \partial Q-\partial Q^{r e g}\right)=0$ for each $x \in S$ and $\alpha \geqq O$, because $G_{0} \geqq G_{\alpha}$. Noting that $\lim _{\alpha \rightarrow+\infty} \alpha \hat{G}_{\alpha} f(x)=f(x)$ uniformly for each $f \in C_{0}$, we can choose $a$ function $f \in C_{0}$ and $\alpha>O$ such that $\hat{G}_{\alpha} f(x)>\delta>O$ on $\partial Q-\partial Q^{r e g}$. Hence it holds that

$$
\begin{aligned}
O & =\int_{S} G_{\alpha}\left(x, \partial Q-\partial Q^{r e g}\right) f(x) m(d x)=\int_{\partial Q-\partial Q^{r e g}} \hat{G}_{\alpha} f(y) m(d y) \\
& \geqq \delta m\left(\partial Q-\partial Q^{r e g}\right),
\end{aligned}
$$

which implies $m\left(\partial Q-\partial Q^{r e g}\right)=0$. Therefore the equality (4) holds $(m)$ almost everywhere. Since the both sides of (4) are $(G, O)$-excessive, the equality holds everywhere. Applying Lemma 3 to the equality (4), we have 


$$
\int_{S} G_{0}(x, z) \hat{P}_{x_{0}}\left(\hat{x}_{\rho_{Q} c} \in d z\right)=\int_{S} G_{0}(x, z) \hat{P}_{x_{0}}\left(\hat{x}_{\sigma_{\partial Q}} \in d z\right), \quad x_{0} \in Q,
$$

for each $x$. Hence by Lemma 4 we have

$$
\hat{P}_{x_{0}}\left(\hat{x}_{\sigma_{Q^{c}}} \in d z\right)=\hat{P}_{x_{0}}\left(\hat{x}_{\sigma_{\partial Q}} \in d z\right), \quad x_{0} \in Q,
$$

which implies (3). We complete the proof.

\section{$\S 5$. Green function and standard processes in the relation of duality}

Let $G(x, y)$ be a Green function on the domain $\Omega \subseteq R^{d}(d \geqq 3)$ in the sense of [2], p. 46, with the condition (S), i.e.,

$$
\frac{C_{1}}{|x-y|^{d-\alpha}} \geqq G(x, y) \geqq \frac{C_{2}}{|x-y|^{d-\alpha}}, \quad x, y \in K, \quad d>\alpha>0,
$$

where $K$ is a compact set in $\Omega$ and $C_{1}, C_{2}$ are strictly positive constants depending only on $K$. We say that $G(x, y)$ is quasi-symmetric, if $G(x, y)$ is continuous in $\Omega \times \Omega$ except on the diagonal set and both $G f(x)$ and $\hat{G} f(x)$ maps $B_{K}$ into $C_{0}$, where $G f(x)=\int_{\Omega} G(x, y) f(y) d y$ and $\hat{G} f(x)=\int_{\Omega} \hat{G}(x, y) f(y) d y$, $\hat{G}(x, y)=G(y, x)$ and further $G$ and $\hat{G}$ satisfy the weak principle of the positive maximum.4)

Lemma 5. For a Green function $G(x, y)$, with the condition $(S)$ in $\Omega$ in the sense of [2], there corresponds a standard process such that

$$
\int_{0}^{ \pm \infty} T_{t} f(x) d t=G f(x), \quad f \in B_{K} .
$$

This lemma is shown in [2].

Proposition. For a quasi-symmetric Green function $G(x, y)$ with the condition $(S)$, there correspond standard processes $X=\left(x_{t}, \zeta, M_{t}, P_{x}\right)$ and $\hat{X}=\left(\hat{x}_{t}, \hat{\zeta}, \hat{M}_{t}, \hat{P}_{x}\right)$ in the relation of duality with respect to Lebesque measure $d x$ such that

$$
\int_{0}^{+\infty} T_{t} f(x) d t=G f(x), \int_{0}^{+\infty} \hat{T}_{t} f(x) d t=\hat{G} f(x), \quad f \in B_{K} .
$$

Further, let $G_{0}(x, y)$ be a kernel for $\alpha=0$ which is constructed in Lemma 2 by setting $m(d x)=d x$, then we have

4) We say that a kernel $G(x, y)$ satisfies the weak principle of the positive maximum if, for a continuous function $f$ of compact support such that $G f \geqq 0, G f$ attains its (strictly positive) maximum at a point of $S$ where $f$ is strictly positive. 


$$
G(x, y)=G_{0}(x, y)
$$

Proof. The existence of such processes follows from Lemma 5. The relation of duality between $X$ and $\hat{X}$ is evident by the definition of $G$ and $\hat{G}$. Hence it is sufficient to show $G_{0}(x, y)=G(x, y)$. Since $G_{0}(x, y)=G(x, y)$ holds $(m)$-almost everywhere for fixed $x(m(d x)=d x)$, we have only to prove $G(x, y)$ is $(\hat{G}, O)$-excessive function of $y$ for each fixed $x .^{5)}$ For each $f \in B_{K}$, $\hat{G} f$ is $(\hat{G}, O)$-excessive, therefore $\alpha \hat{G}_{\alpha} \hat{G} f(x) \leqq \hat{G} f(x)$ for each $\alpha>O$ and hence

$$
\int_{\Omega} \hat{G}(z, y) \alpha \hat{G}_{\alpha}(x, d z) \leqq \hat{G}(x, y)
$$

holds for $(m)$-almost all $y$ for each fixed $x$. As $\hat{G}(x, y)$ is continuous in $y(\neq x)$ and $\int_{\Omega} \hat{G}(z, y) \alpha \hat{G}_{\alpha}(x, d z)$ is lower semicontinuous in $y$, the inequality (5) holds everywhere. On the other hand, if we set $\hat{G}_{n, y}(x)=\min \{\hat{G}(x, y)$ $\wedge n\}$, we have

$$
\lim _{\alpha \rightarrow+\infty} \int_{\Omega} \hat{G}(z, y) \alpha \hat{G}_{\alpha}(x, d z) \geqq \lim _{\alpha \rightarrow+\infty} \int_{\Omega} \hat{G}_{n, y}(z) \alpha \hat{G}_{\alpha}(x, d z)=\hat{G}_{n, y}(x)
$$

By tending $n$ to infinity, we have

$$
\lim _{\alpha \rightarrow+\infty} \int \alpha \hat{G}(z, y) \hat{G}_{\alpha}(x, d z) \geqq \hat{G}(x, y) .
$$

The inequalities (5) and (6) introduce $\lim _{\alpha \rightarrow+\infty} \int_{\Omega} \hat{G}(z, y) \alpha \hat{G}(x, d z)=\hat{G}(x, y)$, which means $G(x, y)$ is a $(\hat{G}, O)$-excessive function of $y$ for each fixed $x$. Thus we have proved the Proposition.

Remark. Also we can prove that $G(x, y)$ is a $(G, O)$-excessive function of $x$ for each fixed $y$ in the same way.

\section{REFERENCES}

[1] G.A. Hunt: Markoff processes and potentials, 3 Illinois J. Math. (1958), 151-213.

[2] M. Kanda: Regular points and Green functions in Markov processes, Soc. Japan, Vol. 19 No. 1, (1967), 46-69.

[ 3 ] H. Kunita and T. Watanabe: Markov processes and Martin boundaries, Illinois J. Math., 9 (1965), 485-526.

5) The following proof is due to Prof. T. Watanabe. 
[4] P.A. Meyer: Semi-groupes en dualité, séminaire Brelot-Choquet-Deny (théorie du potentiel) (1960-1961).

[5] S. Watanabe: On discontinuous additive functionals and Lévy measures of a Markov pröcess, Japanese J. Math. 36 (1964), 53-70.

Nagoya University 